\title{
Viability of HAADF-STEM Imaging Contrast and Simulations as a Measure of B-site Ordering for Double Perovskites
}

\author{
Bryan Esser ${ }^{1}$, Manisha Dixit ${ }^{1}$, Adam Hauser ${ }^{2}$, Robert E A Williams ${ }^{1}$, Fengyuan Yang ${ }^{2}$, Hamish \\ L. Fraser ${ }^{1}$, David McComb ${ }^{1}$ \\ 1. Department of Materials Science and Engineering, The Ohio State University, 477 Watts Hall, \\ 2041 College Road, Columbus, OH 43210, USA \\ 2. Department of Physics, The Ohio State University, 477 Watts Hall, 2041 College Road, \\ Columbus, $\mathrm{OH} 43210$, USA
}

Spintronics has emerged as a promising technology that exploits both the intrinsic spin of the electron and it associated magnetic moment in a solid-state device. Utilization of the spin degree of freedom in metals and semiconductors has potential to create significant technological advances over current, charge-based technologies[1]. Therefore, accurate, atomic level characterization of half-metallic spin injectors and magnetic semiconductors will be essential to realizing these next generation technologies.

A significant barrier to this technological breakthrough was finding materials that exhibit ferromagnetic ordering and spin polarized transport above room temperature. One system that possesses two characteristics needed for practical spintronic applications, the $\mathrm{A}_{2} \mathrm{~B} \mathrm{~B}^{\prime} \mathrm{O}_{6}$ double perovskites (DP), has been studied extensively due to their high spin polarization (P) and Curie temperatures (TC) well above room temperature[2,3]. The fabrication of high-quality DP epitaxial films with high levels of $\mathrm{B} / \mathrm{B}$ ' ordering is essential to achieving desirable spintronic properties, historically however, fabrication and characterization has proven challenging. [4].

High-angle annular dark-field (HAADF) scanning transmission electron microscopy (STEM) image contrast intensity is sensitive to atomic number $(Z)$ and ideal for investigating local atomic arrangements. Therefore, the ability to collect quantitative HAADF images provides potential to collect both chemical and structural information with sub-Angstrom resolution in the time required for a single image acquisition[5]. Quantification of HAADF image contrast in terms of $\mathrm{Z}$ is not trivial, crystalline materials simulated along a zone axis requires separating out the effects of channeling, contributions of inelastic and elastic scattering, and the influence of thermal diffuse scattering and Debye-Waller factors[6]. Recent results show that even for single crystals, image simulations are required to correctly interpret HAADF-STEM image contrast[7].

For this work HAADF-STEM images of a $\mathrm{Sr}_{2} \mathrm{CrReO}_{6}\langle 001<$ film were collected using a FEI probe-corrected HAADF-STEM Titan 80-300 microscope. Imaging along the $\langle 110<$ direction of $\mathrm{Sr}_{2} \mathrm{CrReO}_{6}$ reveals clear variation in column intensity with three distinct levels corresponding to independent atomic columns of $\mathrm{Sr}, \mathrm{Cr}$, and $\mathrm{Re}$. The intensity of each atomic column is proportional to $Z$, for which $\operatorname{Cr}(Z=24$, darkest $), \operatorname{Re}(Z=75$, brightest $)$, and $\operatorname{Sr}(Z=38$, intermediate) were identified. Image comparison and validation were performed against frozenphonon based multi-slice simulation of a $<110>$ SCRO with simulations results exhibiting intensity ratios as expected in the ratio of $\sim Z^{1.8}$. Simulations were also conducted of various Bsite disorder conditions to compare against experimental image intensity variations. Results and comparisons of experimental images to simulated images for quantitative determination of B-site order/disorder will be discussed. 
References:

[1] A. J. Hauser, et al, Physical Review B 85, 161201(R) (2012)

[2] K. L. Kobayashi, et al, Nature 395, 677 (1998).

[3] J. M. Serrate, De Teresa, and M. R. Ibarra, J. Phys.: Condens. Matter 19, 023201 (2007).

[4] G. Blamire, et al, Adv. Mater. 21, 3827 (2009).

[5] J. M. LeBeau and S. Stemmer, Ultramicroscopy 108, 1653 (2008)

[6] S.D. Findlay, et al, Institute of Materials, Minerals and Mining, (2008)

[7] J.M. Lebeau, et al, Physical Review B 79, 214110 (2009)

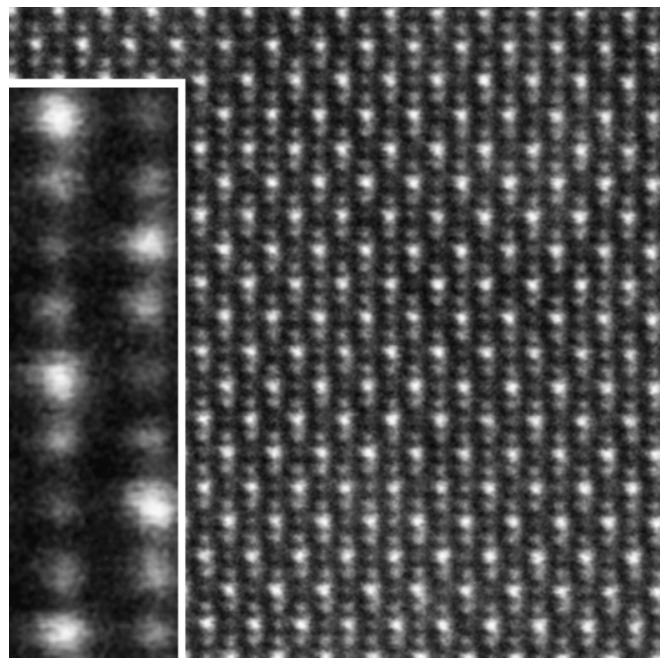

Fig 1: HAADF-STEM image of $<110>$ SCRO exhibiting Z-contrast.
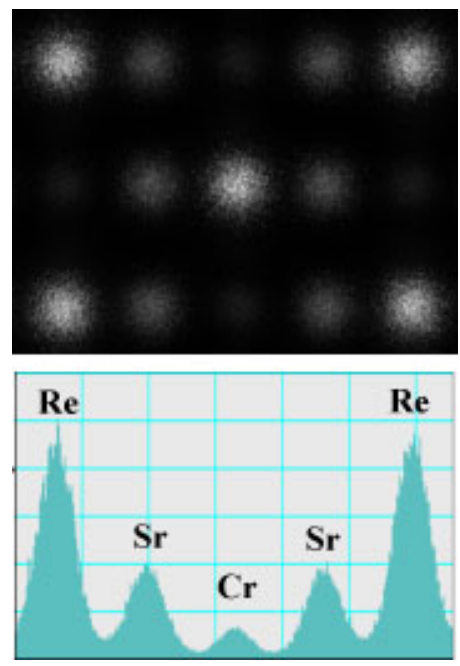

Fig 2: HAADF-STEM multislice image simulation of $<110>$ SCRO showing ideal column intensity ratios.

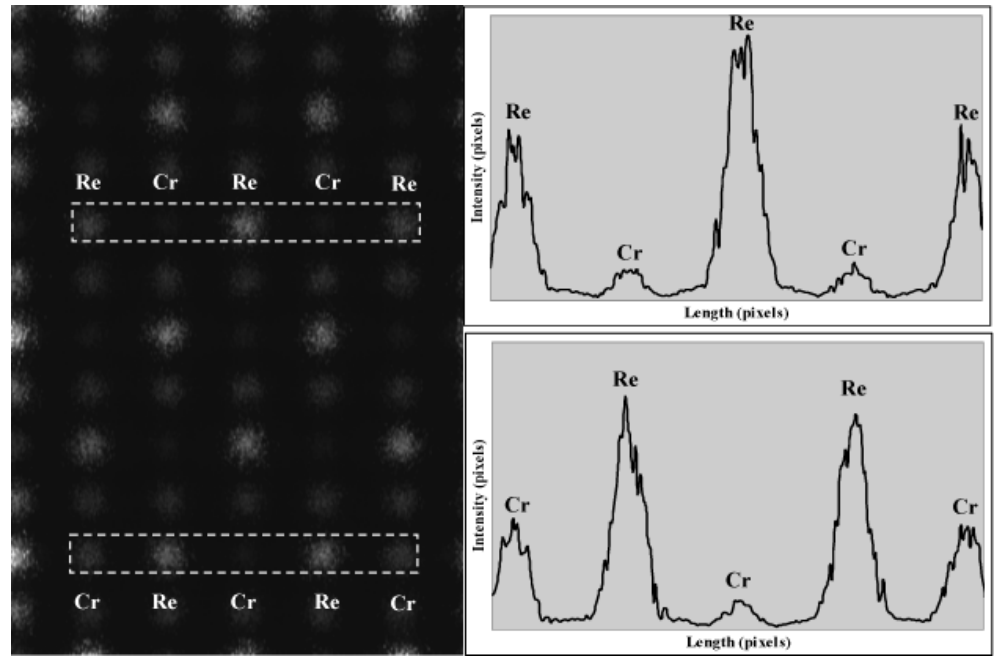

Fig 3: HAADF-STEM multislice image simulation of $<110>$ SCRO showing non-stoichiometric column intensity resultant from B-site disorder. 